\title{
Philosophiques
}

\section{Introduction générale : l’impasse des égaux}

\section{Louise Marcil-Lacoste}

Volume 11, numéro 1, avril 1984

URI : https://id.erudit.org/iderudit/203244ar

DOI : https://doi.org/10.7202/203244ar

Aller au sommaire du numéro

\section{Éditeur(s)}

Société de philosophie du Québec

ISSN

0316-2923 (imprimé)

1492-1391 (numérique)

Découvrir la revue

\section{Citer cet article}

Marcil-Lacoste, L. (1984). Introduction générale : l'impasse des égaux. Philosophiques, 11(1), 113-123. https://doi.org/10.7202/203244ar

\section{Résumé de l'article}

Présentant les textes publiés dans ce numéro de Philosophiques comme résultats préliminaires d'une recherche collective sur les théories de l'égalité et les problèmes philosophiques de la condition féminine, l'auteur soutient que leur contribution majeure réside dans une triple réévaluation des enjeux relatifs à l'égalité : le parallélisme étanche qui caractérise en ce moment les recherches sur l'égalité d'une part et les recherches sur la condition féminine d'autre part doit être corrigé; une démarche post-rawlsienne reliant la question de l'égalité à un traitement équitable des multiples formes de différences se manifestant entre les humains est grandement nécessaire; les limites caractérisant les plaidoyers contemporains en faveur d'une égalité par-delà les différences doivent être identifiées. 


\title{
INTRODUCTION GÉNÉRALE : L'IMPASSE DES ÉGAUX
}

\author{
par Louise Marcil-Lacoste
}

\begin{abstract}
RÉSUMÉ. Présentant les textes publiés dans ce numéro de Philosophiques comme résultats préliminaires d'une recherche collective sur les théories de l'égalité et les problèmes philosophiques de la condition féminine, l'auteur soutient que leur contribution majeure réside dans une triple réévaluation des enjeux relatifs à l'égalité : le parallélisme étanche qui caractérise en ce moment les recherches sur l'égalité d'une part et les recherches sur la condition féminine d'autre part doit être corrigé ; une démarche post-rawlsienne reliant la question de l'égalité à un traitement équitable des multiples formes de différences se manifestant entre les humains est grandement nécessaire ; les limites caractérisant les plaidoyers contemporains en faveur d'une égalité par-delà les différences doivent être identifiées.
\end{abstract}

ABSTRACT. Presenting the papers included in this issue of $\mathrm{Pbi}$ losophiques as the preliminary results of a three years research project on theories of equality and philosophical problems of the women condition, it is argued that their main contribution lies in a threefold reasessment of the issues of equality : the impervious parting which presently caracterizes researches on equality, on the one hand, and researches on women, on the other hand, must be corrected ; a post-rawlsian approach relating the question of equality to a just treatment of manifold differentiations among human beings is highly needed ; and the shortcomings of present pleas in favor of an equality beyond differentiations must be identified.

L'ensemble des enquêtes dont nous présentons ici les premiers résultats relèvent d'une recherche collective sur " Les théories de l'égalité et les problèmes philosophiques de la condition féminine » menée au Département de philosophie de l'Univesité de Montréal. Subventionnée par le Conseil de recherche en sciences humaines du Canada et par le fonds de la Formation 
de chercheurs et action concertée du Ministère de l'éducation du Québec pour une troisième année consécutive, cette recherche a porté sur le premier volet de la question à l'étude au cours de sa première année d'activités, c'est-à-dire, pour l'année 19811982, sur les théories de l'égalité.

Les études présentées ici abordent donc toutes la question de l'égalité dans son sens général. Plusieurs font allusion au problème de la condition féminine, mais c'est toujours dans le but de faire ressortir l'enjeu fondamental d'une théorie générale de l'égalité. L'accent que l'on met ici sur la notion d'égalité, avant et dans le but d'aborder la question des rapports entre les hommes et les femmes étonnera sans doute. Il est devenu courant, en effet, de dissocier les réflexions dites " générales » sur l'égalité et les réflexions dites «spécialisées ", voire "féministes ", sur la condition féminine. Lorsqu'on parle d'égalité, on discute rarement du « cas » de la femme. À l'inverse, lorsqu'on parle de la condition féminine, on discute rarement de la thématique contemporaine de l'égalité.

L'équipe de recherche tient cette dissociation pour un indice supplémentaire des problèmes philosophiques marquant l'analyse de la condition féminine. Il en résulte bien des malentendus, le plus fréquent, peut-être, étant la facilité avec laquelle on aborde ou conclut une étude sur la condition féminine en faisant référence, voire en faisant appel à un idéal d'égalité qui aurait les propriétés de l'idée claire et du fondement éthique reconnu. Mais, pour dire les choses promptement, s'il est une idée dont la popularité a crû en proportion directe de son ambiguïté et des controverses qu'elle suscite, l'égalité pourrait bien en être le paradigme contemporain.

Cette dissociation heuristique entre l'égalité et le féminin donne lieu à de nombreuses méprises. Par exemple, on imagine souvent qu'il suffit de rapprocher les problématiques pour régler les problèmes du féminin par les solutions des théories de l'égalité. Autre leurre. Les théories de l'égalité offrent de si nombreux exemples de doctrines anti-égalitaires qu'on ne voit pas comment et par quelle magie épistémologique leur simple « application » au cas de la femme pourrait contribuer à l'amélioration de la condition féminine. 
Il devient dès lors urgent d'analyser les paramètres généraux des théories de l'égalité, même lorsqu'il s'agit, en l'espèce, de commencer à apercevoir l'horizon d'une solution dans le " cas" de la femme. Ces paramètres appellent des modifications " générales » des théories, avant même que l'on puisse songer à l'émergence de telles solutions.

En bref, toutes les études présentées ici reposent sur le refus de dissocier la question de la femme de la question de l'égalité. Elles reconnaissent l'urgence de situer le débat philosophique sur la femme dans le bastion théorique qui, de manière souterraine mais perceptible, en définit le cadre, les limites, les impasses, les horizons : les débats sur l'égalité.

\section{UNE APPROCHE POST-RAWLSIENNE}

Les réflexions que nous présentons ici sur l'égalité se situent à un carrefour particulièrement pertinent et controversé d'analyses, dans la mesure où elles abordent les rapports entre l'égalité et la justice, d'une part, et l'égalité et la différence, d'autre part. On peut, en effet, en décrire la facture comme étant post-rawlsienne. À la suite de Rawls et depuis la parution de A Theory of Justice (1970), il est devenu urgent de re-penser les rapports entre les deux termes les plus controversés de la devise de la Révolution française, à savoir la liberté et l'égalité. En l'occurrence, il est devenu crucial de s'interroger désormais sur les fameuses "différences » dont se sont nourris les défenseurs de la liberté et les pourfendeurs de l'égalité.

On peut, dès lors, qualifier de " post-rawlsienne " toute programmatique qui s'interroge sur le statut épistémologique, axiologique, politique des différences entre individus, groupes et ensembles sociétaux, à la lumière ou dans l'horizon de la justice, ou plus précisément à la lumière d'une nouvelle définition du contrat social. L'originalité de cette perspective tient au renversement de perspective quant à l'enjeu égalitaire de la question des différences. Au lieu de présumer que les différences sont soit irréductibles, soit irrecevables, au lieu de présumer que leur existence même témoigne de la primauté de la liberté sur l'égalité, dans tous les cas où il y a conflit entre ces deux valeurs, on tente d'établir que les différences ne relèvent pas d'un lieu, 
ni d'un statut, ni d'une prégnance identiques (les unes sont recevables, les autres pas ; les unes sont modifiables, les autres irréductibles, etc.). En outre, on tente d'établir que l'existence même de ce réseau de différences ne témoigne ni de la primauté de la liberté sur l'égalité (solution "libérale " du conflit entre ces valeurs), ni de la primauté de l'égalité sur la liberté (solution " socialiste " du conflit entre la liberté et l'égalité), mais plutôt de la primauté heuristique d'une re-définition de la justice.

Mais suffit-il ici de renvoyer la liberté et l'égalité dos à dos au tribunal de la justice, voire de l'équité, pour réaliser la promesse égalitaire que recèle ce renversement de perspective ? Jusqu'ici, semble-t-il, les analystes se sont partagés le fardeau de l'insatisfaction, en recourant aux classifications courantes : les uns trouvent que la thèse de Rawls est trop libérale, les autres, trop socialisante. Peu ont tenté de reprendre la question à sa source : qu'en est-il des rapports entre l'égalité et la justice? Dans quelle mesure une pensée de la différence peut-elle s'allier à une visée égalitaire?

L'ensemble des études présentées ici constituent autant d'enquêtes sur diverses facettes de ces questions capitales. Toutes s'intéressent au statut effectif que l'on accorde à l'une ou l'autre des différences par lesquelles on pense la justice, la hiérarchie, l'équivalence, la démocratie, la liberté. Certaines études se réferent surtout à un ou des auteurs (Aristote, Hegel, Harding, Montrelay). D'autres s'intéressent à un ensemble d'écrits sur la question retenue, offrant une synthèse partielle. Les unes mettent l'accent sur le diagnostic : l'irruption de l'inégalité dans telle ou telle problématique. D'autres mettent l'accent sur la prospective : le projet égalitaire, ses conditions d'émergence. Toutes se refusent aux aprioris péremptoires qui n'annonceraient de solution que pour avoir fait l'économie des problèmes. Chacune espère contribuer au déblayage, voire au déblocage de la logique par laquelle, jusqu'ici, il a été à peu près impossible de penser l'enjeu du féminin sur le terrain même où la philosophie a confiné la "question " de la femme : l'impasse des égaux.

\section{LES IMPASSES}

Car c'est bien en parlant d'impasses qu'il convient de décrire les résultats heuristiques provisoires auxquels cette première année de recherche sur l'égalité nous a conduit. Qu'il s'agisse en effet 
des études d'ensemble ou des études thématiques, chacune permet de déceler le noyau épistémique, axiologique ou historique qui explique soit une facette, soit une origine des piétinements qui caractérisent les réflexions contemporaines sur l'égalité.

On aura une première idée, assez générale, de tels piétinements en prenant connaissance de ce que j'ai appelé la " typique » contemporaine de l'égalité, c'est-à-dire les caractéristiques majeures des écrits théoriques consacrés à l'égalité au $\mathrm{XX}^{\mathrm{e}}$ siècle. Dans mon étude consacrée aux « Cent quarante manières d'être égaux », j'analyse en effet les multiples désignations dont ia notion contemporaine d'égalité est chargée. Ce qui, à première vue, ne semblerait peut-être qu'une comptabilité mécanique, se révèle, après analyse, le fondement d'un des diagnostics le plus percutant que l'on puisse objectivement établir concernant la thématique contemporaine : en privilégiant, d'une manière systématique, une notion univoque assez étroite de l'égalité, les théoriciens du $\mathrm{XX}^{\mathrm{e}}$ siècle tissent une problématique relativement répétitive où le privilège que l'on accorde à certaines impasses théoriques (par exemple, le gouffre entre l'égalité " générale » et les égalités "spécifiques », entre l'égalité de droit et l'égalité de fait, entre l'égalité comme idéal ou comme processus historique) contrevient directement à l'armature conceptuelle effectivement élaborée depuis un demi-siècle, son foisonnement, ses spécifications, ses relais multi-dimensionnels, ses assises historiques. En un mot, on continue de théoriser sur l'égalité comme si la notion intuitive et anhistorique de chacun pouvait rendre compte de toutes les promesses et de toutes les impasses égalitaires, lesquelles à leur tour traduiraient adéquatement la structure, voire la méta-structure, des relations interindividuelles, groupales et systémiques caractérisant la société actuelle.

C'est justement ce genre d'a priori que les études présentées ici mettent en cause, dans le but notamment de procéder à une définition du problème de l'égalité qui se dégagerait des ornières et des visières par trop courantes. Les membres de l'équipe ayant eu l'occasion d'analyser et de discuter de diverses notions de l'égalité, ainsi que de diverses manières d'en rendre compte sur un plan typologique, on peut traduire leur souci commun de la manière suivante : si, comme je le montre dans les « Cent qua- 
rante manières d'être égaux ", il existe une telle richesse conceptuelle et théorétique dans la thématique contemporaine de l'égalité, alors comment expliquer les piétinements des projets égalitaires?

À ce niveau de questionnement, les avenues à explorer deviennent plus spécifiques encore et, en vertu de cette nouvelle spécification, l'énoncé des diagnostics devient plus précis et plus dialectique. Dans la plupart des cas, du reste, ces diagnostics ont une facture historique qui a l'avantage de situer sur terre les débats formels auxquels la pensée contemporaine nous a habitués concernant l'égalité.

\section{ÉGALITÉ ET JUSTICE}

On nous a habitués, par exemple, à traiter des rapports entre l'égalité et la justice d'une manière intuitive et sommaire. Les uns opposent les deux termes : l'égalité à tout prix, dira-ton, contrevient à une juste distribution des biens, des statuts, des pouvoirs. Les autres, plus nombreux, associent si étroitement la notion d'égalité et la notion de justice (l'égalité, dira-t-on, n'a de valeur axiologique que dans la mesure où l'on peut la déduire de la justice, voire l'identifier à elle) que les controverses sur l'égalité perdent toute spécificité, voire tout intérêt. Dans les études présentées ici, chacune de ces thèses opposées fait l'objet d'analyses permettant de reformuler les termes de l'opposition entre l'égalité et la justice (Jocelyn Beausoleil) et de mettre en cause les termes de leur identification (Jocelyne StArnaud).

Peu ont étudié le paradoxe de la notion contemporaine de l'égalité à la lumière de son irréductibilité par rapport à une justice dont elle serait néanmoins le schème. C'est une telle analyse que nous offre ici Jocelyn Beausoleil, dans son étude systématique de diverses définitions contemporaines de l'égalité intitulée "À quoi peut bien servir la notion d'égalité, si elle n'est vraiment heuristique, ni simplement éristique ? ». Jocelyn Beausoleil explique le paradoxe de l'égalité dans la société industrielle (le fait que cette société soit égalitaire dans ses aspirations et hiérarchique dans son organisation) à la lumière des ambiguïtés que recèle la genèse de l'idée d'égalité depuis le XVIII 
siècle. Ceci lui permet de mettre en évidence le caractère fondamentalement vide du concept d'égalité qui en explique la fluctuation culturelle et, du même coup, l'irréductibilité par rapport à la justice.

Par ailleurs, lorsqu'on parle des rapports entre la justice et l'égalité dans une perspective visant à leur identification, on évoque souvent les thèses classiques d'Aristote. Or, c'est ce que Jocelyne St-Arnaud montre bien dans son étude « Les définitions aristotéliciennes de la justice, Leurs rapports à l'égalité », quand on relie les diverses notions de la justice du Livre V de l'Éthique à Nicomaque aux deux notions d'égalité systématisées par Aristote, c'est-à-dire l'égalité arithmétique et l'égalité géométrique, on peut établir que la notion aristotélicienne de justice distributive est foncièrement inégalitaire, tandis que sa notion de justice directive est celle d'une égalité formelle. Jocelyne StArnaud conclut son analyse en soutenant qu'Aristote a ainsi fourni les deux schèmes explicateurs de tout partage des biens et du pouvoir, un schème proportionnel et un schème égalitaire. Quand on prend connaissance de ces deux schèmes et de leur prégnance dans les débats contemporains, notamment dans les controverses sur les rapports entre l'égalité et la méritocratie, on comprend du même coup en quel sens il est facile de condamner les égaux à l'impasse : il suffit de jouer diverses définitions de la justice les unes contre les autres.

Ajoutons que lorsqu'on aborde la question des rapports entre la justice et l'égalité en songeant à l'enjeu des hiérarchies sociales, c'est-à-dire à la question qui tisse une armature idéologique centrale au $\mathrm{XX}^{\mathrm{e}}$ siècle, on prend aisément pour acquis qu'il suffit de compléter une théorie sociale de la justice par une programmatique démocratique pour pallier les impasses des égaux. Pourtant, c'est ce que montre l'étude de Christiane Gohier, la notion de démocratie n'est pas nécessairement égalitaire. En proposant « Le rapport masse/élite comme modèle canonique de la dialectique sociale ", Christiane Gohier recourt à la mesure de l'égalité sociale proposée par Oppenheim. Cet étalon lui permet d'évoquer diverses formes d'inégalités sociales (dominant/ dominé, seigneur/serf, maître/esclave, etc.) pour établir la structure foncièrement inégalitaire des sociétés libérales et socialistes. Derrière les paradigmes connus de l'inégalité et dont le " maître 
et l'esclave " constituent l'exemple le plus fréquemment étudié, on peut discerner l'existence d'un invariant structurel : le rapport masse/élite. Ce rapport a la double fonction de désigner un inférieur par rapport à un supérieur et une grande majorité d'individus par rapport à quelques personnes. Cette double structure du rapport de domination fait apparaître le caractère non-nécessairement égalitaire de la notion même de démocratie.

\section{ÉGALITÉ ET DIFFÉRENCE}

D'une manière non moins significative, les poncifs de la pensée contemporaine sur les rapports entre l'égalité et « la différence » sont ici mis en cause. Tout d'abord, en effet, ce paradoxe immense et d'une prégnance apparemment à toute épreuve : chacun proteste du fait que l'égalité n'est pas synonyme d'identité, tout en attribuant cependant cette thèse à toute théorie égalitaire, si bien qu'en apparence, seule l'égalité revendiquée tomberait dans le gouffre identitaire. Peu s'interrogent, cependant, sur la manière dont l'obsession de l'identique traverse les discours inégalitaires et les discours sur la différence, comme si les différences n'étaient pensables qu'en incantations, voire en rhétorique anti-identitaires. $\mathrm{Si}$, comme chacun le répète, l'égalité ne saurait être synonyme d'identité, il est cependant loin d'être évident que l'égalité puisse s'allier à " la différence".

L'obsession de l'identique a donc plusieurs visages sur lesquels les études présentées ici permettent de faire le point. Par exemple, on attribue aisément à une influence logico-mathématique abusive la double idée que l'égalité serait synonyme d'identité et, par conséquent, formellement inapplicable. Cette double allégation, on le verra en prenant connaissance de la note d'Yvon Gauthier, est fausse au moins de deux manières. Tout d'abord, même en logique, la notion d'égalité a une structure formelle plus riche que le seul relais identitaire. En outre, c'est en réduisant la logique à une stratégie identitaire, elle-même amputée de ses dispositifs les plus prometteurs, que l'on fait de la logique le bouc émissaire des impasses égalitaires, tout en lui refusant la possibilité de contribuer, d'une manière prospective, à leur solution. Dans "Syntaxe et sémantique du concept d'égalité », Yvon Gauthier met donc à jour la structure logico-formelle du concept d'égalité, distinguant l'égalité extensionnelle 
et l'égalité intensionnelle et rejetant le concept d'identité comme relais de la notion d'égalité. Établissant un constat d'échec quant à l'idéal rationnel d'équivalence, Yvon Gauthier propose une alternative fondée sur une distinction entre les propriétés homotopiques et hétérotopiques.

Du reste, faut-il le souligner, l'idée que seule la logique serait ici en cause est bien courte. C'est toute la philosophie, la métaphysique et la morale de l'Un, du Même et de l'Homogène qui est en procès, celles-là mêmes que, paradoxalement, on invoque pour fustiger les projets égalitaires. Car si plusieurs se sont intéressés à la déconstruction du «Même », bien peu ont tenté de formuler explicitement ce qui pourrait être une philosophie égalitaire de l'Autre. Jacques-Bernard Roumanès s'y emploie ici, en proposant "Une alternative à l'exclusion : la dialogique ». Expliquant la dynamique du système institutionnel comme le résultat de la tentative de « tout ramener au même », Jacques-Bernard Roumanès propose l'alternative d'une dynamique d'échanges alternatifs entre partenaires réels. Se demandant si une démocratie non-anarchiste est possible, il justifie sa préférence pour la dynamique des échanges alternatifs. Le cercle vicieux d'une position logique univoque et hiérarchique doit être remplacé par les confrontations d'une dia-logique donnant une position théorique valable à l'altérité. Toute la question consiste à savoir dans quelle mesure il est possible de baliser le passage d'une égalité identitaire à une égalité surgie du respect des différences.

Pour plusieurs, à ce stade de l'analyse, le discours sur la différence n'est qu'une version moderne de plaidoyer libéral opposant l'égalité et la liberté. Dans son étude consacrée à Hegel et intitulée « De la société civile à l'État : l'irruption de l'inégalité », France Giroux éclaire certaines racines de ce débat en mettant en évidence la nature des critiques qu'une pensée dite libérale, voire conservatrice, peut adresser au libéralisme politique issu de la Révolution française, situant ainsi d'une manière plus précise la nature de l'inégalité proclamée. Le passage de la société civile à l'État marque, en effet, dans la philosophie hégélienne l'émergence de l'inégalité politique (entre les sujets et les législateurs ou fonctionnaires) et de l'inégalité sociale (les exclus de la Déclaration des droits, c'est-à-dire la classe ouvrière). Le 
diagnostic que pose Hegel sur la Révolution française permet de situer ses thèses entre celles de Platon et de Rousseau ; il permet surtout de désigner l'inadéquation d'une présentation de la philosophie hégélienne soit comme philosophie libérale, soit comme philosophie conservatrice.

Dans la pensée contemporaine, enfin, on prend trop facilement pour acquis que la désirabilité d'une égalité dans le respect des différences témoignerait de sa possibilité. Or, rien n'est moins sûr. L'impasse des égaux traverse si aisément les discours sur la différence qu'il faut plutôt conclure, avec Michèle Morosoli, à la nécessité d'une déconstruction non seulement de l'Un mais aussi du Multiple, non seulement du Même, mais aussi de l'Autre, tous deux, dans leur nomination inégalitaire.

Dans "Les avatars de la différence ", Michèle Morosoli montre en effet à quel point certains discours psychanalytiques sur l'homme et la femme proposent, au nom même de la différence, un double mouvement d'exclusion de la différence et de sa ré-inclusion comme contraire. Le coefficient inégalitaire des discours sur la différence se rattache ainsi à leur dialectique d'oppositions hiérarchiques. À leur tour, ces coefficients d'inégalité expliquent le décalage entre l'égalité « générale » et les égalités "spécifiques ", les secondes étant plus quantifiables et applicables que les premières.

Cette manière d'interroger l'égalité "générale » à partir des discours sur les différences tranche avec le scénario courant où les égalités "spécifiques » sont traitées comme autant de régionalismes épistémologiques. En réalité, ce décalage principiel que l'on établit entre l'égalité " générale » et les égalités «spécifiques » explique à lui seul le terrain sur lequel surgit et s'alimente l'impasse des égaux.

Au plan méthodologique, ou à tout le moins programmatique, nos premières enquêtes sur l'égalité nous permettent donc d'inverser, dorénavant, les présupposés. Au lieu de poser l'immunité axiologique des théories générales de l'égalité, partant, au lieu d'expliquer les inégalités effectives par la nonapplicabilité des théories générales en vertu des aléas de l'histoire, on posera plutôt l'efficacité historique considérable des théories générales de l'égalité dans la justification même des inégalités 
spécifiques. Pour paraphraser, mais sans orthodoxie, le mot de Hegel sur ceux qui auraient les mains pures, si ils avaient des mains, on pourrait dire que les discours contemporains sur l'égalité ont bel et bien des mains, mais des mains inégalitaires et que c'est la raison pour laquelle il est plus commode de les déclarer manchots.

En d'autres termes, à cette phase-ci de nos recherches, nous pouvons établir que les piétinements de la thématique contemporaine de l'égalité ne sauraient faire l'objet d'une analyse adéquate à moins d'une rupture systématique avec la prédilection contemporaine pour une égalité procédurale, cette forme d'égalité dont le seul formalisme réside dans sa justification du silence sur les obstacles spécifiques qui jalonnent les processus d'égalisation.

Après avoir montré comment les théories générales de l'égalité peuvent rendre compte des impasses des égaux, il reste dès lors à étudier comment et à quelles conditions les rapports spécifiques entre les deux moitiés du monde, c'est-à-dire les hommes et les femmes, pourraient laisser émerger des théories égalitaires. Il reste, en somme, à répondre à une question que nous posions au début de cette recherche, à savoir : comment une pensée de la différence peut-elle s'allier à une visée égalitaire?

C'est à cette tâche que l'équipe de recherche consacre dorénavant ses activités. Entre temps, nous espérons avoir au moins démontré que telle était désormais la question à poser.

Département de philosophie Université de Montréal 